\title{
ERRATUM
}

G. T. Pecl • N. A. Moltschaniwskyj • S. R. Tracey •

A. R. Jordan

\section{Inter-annual plasticity of squid life history and population structure: ecological and management implications}

\section{Oecologia (2004) 139:515-524}

In the pdf and print versions of this paper, the second half of Table 2 was missing. The html version is correct.

Table 2 Annual sea surface temperature for Great Oyster Bay, Tasmania for each of the sampling years, and estimated average temperature at hatching for the individuals comprising each year's spawning population. A summary of the biological parameters are also shown

\begin{tabular}{|c|c|c|c|c|c|c|}
\hline \multicolumn{2}{|l|}{ Annual temperature } & 1995 & 1996 & 1999 & 2000 & 2001 \\
\hline \multicolumn{2}{|l|}{ Mean location $\left({ }^{\circ} \mathrm{C}\right)$} & 14.45 & 14.11 & 15.67 & 15.52 & 15.86 \\
\hline \multicolumn{2}{|l|}{ Amplitude } & 2.61 & 1.95 & 2.60 & 2.55 & 2.19 \\
\hline \multicolumn{2}{|l|}{ Phase } & 349.42 & 354.29 & 334.85 & 329.22 & 358.06 \\
\hline \multicolumn{2}{|l|}{ Cycle } & 67.82 & 64.79 & 65.52 & 55.10 & 70.43 \\
\hline \multicolumn{2}{|l|}{ SSQ temperature curve } & 14.16 & 6.35 & 13.12 & 12.83 & 10.40 \\
\hline \multicolumn{2}{|c|}{ Hatching temperature average $\left({ }^{\circ} \mathrm{C}\right)$} & 12.17 & 14.40 & 15.51 & 16.15 & 15.91 \\
\hline \multicolumn{2}{|l|}{ Biological parameters } & January 1995 & $1996 / 1997$ & $1999 / 2000$ & $2000 / 2001$ & $2001 / 2002$ \\
\hline \multicolumn{2}{|c|}{ Age-weight overall correlation } & 0.852 & 0.708 & 0.191 & 0.425 & NA \\
\hline \multirow[t]{2}{*}{ Average age (days) } & Males & 149 & 179 & 198 & 188 & NA \\
\hline & Females & 147 & 168 & 191 & 194 & NA \\
\hline \multirow[t]{2}{*}{ Average size (g) } & Males & 482 & 688 & 873 & 1,014 & 1,175 \\
\hline & Females & 444 & 523 & 752 & 637 & 625 \\
\hline \multirow[t]{2}{*}{ Condition } & Males & Good & Average & Average & Good & Poor \\
\hline & Females & Good & Average & Average & Poor & Average \\
\hline \multirow[t]{2}{*}{ Reproductive investment } & Males & Average & Good & Average & Average & Average \\
\hline & Females & Average & Good & Poor & Poor & Poor \\
\hline \multicolumn{2}{|l|}{$\%$ Females } & 51.5 & 41.3 & 33.2 & 20.4 & 35.3 \\
\hline \multicolumn{2}{|l|}{ Sexual dimorphism } & None & Minor & None & High & Extreme \\
\hline
\end{tabular}

The online version of the original article can be found at http://dx. doi.org/10.1007/s00442-004-1537-z

G. T. Pecl $(\bowtie) \cdot$ S. R. Tracey · A. R. Jordan

Marine Research Laboratories, Tasmanian Aquaculture and

Fisheries Institute, University of Tasmania,

Private Bag 49, 7001 Hobart, Tasmania, Australia

e-mail: Gretta.Pecl@utas.edu.au

Fax: +61-3-62278035

N. A. Moltschaniwskyj

School of Aquaculture, Tasmanian Aquaculture and Fisheries

Institute, University of Tasmania,

Locked Bag 1370, 7250 Launceston, Tasmania, Australia 